\title{
Ways to Improve the Efficiency and Reliability of RF Driven Negative Ion Sources for Fusion ${ }^{\text {a) }}$
}

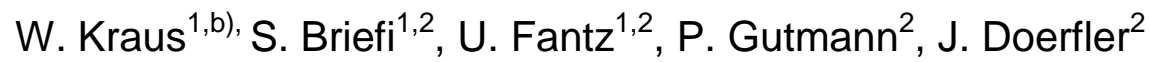 \\ ${ }^{1}$ Max-Planck-Institut für Plasmaphysik(IPP), Boltzmannstr. 2, 85748 Garching, Germany \\ ${ }^{2}$ AG Experimentelle Plasmaphysik, Universität Augsburg, 86135 Augsburg, Germany
}

\begin{abstract}
Large RF driven negative hydrogen ion sources are being developed at IPP Garching for the future NBI system of ITER. The overall power efficiency of these sources is low, because for the RF power supply self-excited generators are utilized and the plasma is generated in small cylindrical sources ("drivers") and expands into the source main volume. At IPP experiments to reduce the primary power and the RF power required for the plasma production are performed in two ways: The oscillator generator of the prototype source has been replaced by a transistorized RF transmitter and two alternative driver concepts, a spiral coil, in which the field is concentrated by ferrites, which omits the losses by plasma expansion and a helicon source are being tested.
\end{abstract}

\section{INTRODUCTION}

The powerful RF ion sources used in the IPP testbeds, in neutral beam injection systems (NBI) of ASDEXUpgrade, of W7X and in future of ITER are till now supplied by self-excited RF generators with one or two tubes (triodes or tetrodes). Common to all these generators is a low power efficiency of $<60 \%$. For example in the ITER NBI four $200 \mathrm{~kW} / 1 \mathrm{Mhz}$ generators will supply each source, requiring a primary power of approx. 1.4 MW and a corresponding water cooling system for $600 \mathrm{~kW}^{1}$. Other drawbacks of oscillators are the dependency on the availability of the tubes, which may be a problem in future, and difficult matching due to the changing oscillator frequency. These problems could be omitted by the use of solid state based generators, which have replaced the tube generators in the field of radio broadcast since many years. These generators have an efficiency of approx. $90 \%$ and do not require water cooling. Because the frequency is not affected by the load, the matching is expected to be less difficult. The self-excited generators for fusion sources are individually designed prototypes, whereas for the solid state generators well-designed reliable series products are available. Because of these potential advantages, a proof of principle test of the applicability of such a type of generator for the supply of powerful RF ion sources is being carried out at IPP.

The production of hydrogen ions and atoms in separate drivers followed by expansion into the source is also suspected to be not very efficient. For each of the drivers which have a volume of only $6 \mathrm{l}$ a power of $70-80$ $\mathrm{kW}$ is needed to achieve the maximum negative hydrogen ion current ${ }^{2}$. It is at least questionable, if in long pulses or in cw operation a reliable source operation can be obtained at such a high power density, which is close to the limit of

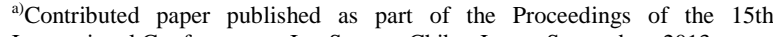
International Conference on Ion Source, Chiba, Japan, September, 2013.

${ }^{\text {b) }}$ Corresponding Author: Werner Kraus, e-mail address: kraus@ipp.mpg.de
}

what the source can stand. A reduction to about $50 \mathrm{~kW}$ would relax the situation substantially. At this power level one hour pulses already have been demonstrated ${ }^{3}$. This was the motivation to investigate other potentially more efficient driver concepts. The first is a flat spiral coil, which omits the driver volume and the plasma expansion, the second is a Helicon coupling. First experiments with these sources on small test facilities are reported.

\section{TRANSISTOR GENERATOR}

In the self-excited generators used at IPP the tank circuit is supplied by the anode voltage of $12 \mathrm{kV}$ and the power is coupled to the transmission line by an output transformer. The maximum power is $100-180 \mathrm{~kW}$ at 1 $\mathrm{MHz}$. The matching is done by a variable capacitor switched in series to the RF coil. For the variation of the frequency additional variable capacitors in the tank circuit are needed.

At the Batman test facility ${ }^{2}$ the $100 \mathrm{~kW} / 1 \mathrm{MHz}$ oscillator, which supplies the negative ion source, was replaced by a $75 \mathrm{~kW}$ transistorized radio transmitter of TRANSRADIO SenderSysteme Berlin AG. This generator was originally designed as a $50 \mathrm{~kW}$ AM broadcast transmitter and has been modified according to the requirements of the plasma load. It consists of 50 modules of maximal $1.5 \mathrm{~kW}$ in which a voltage of up to $260 \mathrm{~V}$ is switched by four transistors. The resulting square wave voltages are arranged in parallel on a "combiner" ferrite core and transformed into a sinusoidal wave by an output filter. Due to the modular concept the generator is still operable, when one or more modules fail. Each of the modules can be exchanged in a short time. More powerful generators up to $2 \mathrm{MW}$ consist of several of these $75 \mathrm{~kW}$ units. For the tests with the plasma load the maximum tolerable SWR (standing wave ratio) was adjusted to 2, corresponding to a load between $25 \Omega$ and $90 \Omega$. In order to enable frequency matching, the frequency can be varied by $+/-50 \mathrm{kHz}$. 
In the RF source the power is inductively coupled into a circular volume of $25 \mathrm{~cm}$ diameter, out of which the plasma is flowing into the main chamber (b x l x d = $30 \mathrm{x}$ $59 \times 25 \mathrm{~cm}^{3}$ ). The design is described in detail in ${ }^{4}$. The ITER source will have eight of these "drivers". The Batman source is operated in a duty cycle of $6 \mathrm{~s}$ pulse/ 3 min interruption. To ignite the discharge a small starter filament and a short pressure pulse is necessary.

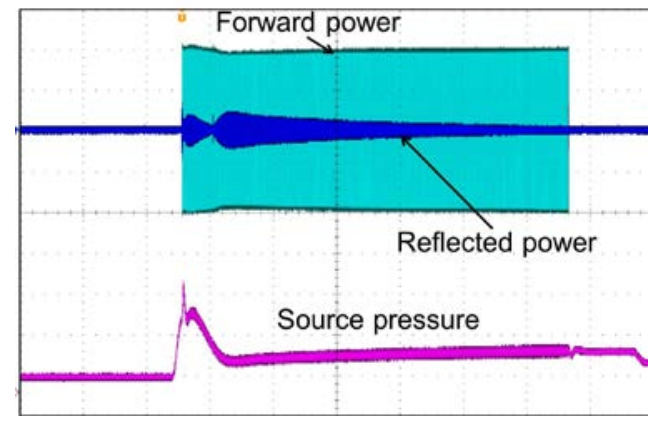

FIG.1. A 6 s plasma pulse with an RF power of $60 \mathrm{~kW}$; shown is the voltage proportional to the forward and to the reflected power and the source pressure. The pulse starts at $1.5 \mathrm{~Pa}$ and ends at appox 0.6 Pa.

The transistor generator has been connected to the same matching circuit as the oscillator used before. Plasma ignition is only possible in a narrow capacity range of about $100 \mathrm{pF}$. After the matching to the plasma load is completed, operation up to the maximum power of $75 \mathrm{~kW}$ is possible. The pressure in the source varies between 1.5 $\mathrm{Pa}$ during the start phase and $0.4-0.6 \mathrm{~Pa}$ during source operation. The SWR is not constant during the pulse, because the reflected power depends on the source pressure. But it is possible to match close to SWR $=1$ (no reflected power) in the second half of the pulse (see Fig.1).

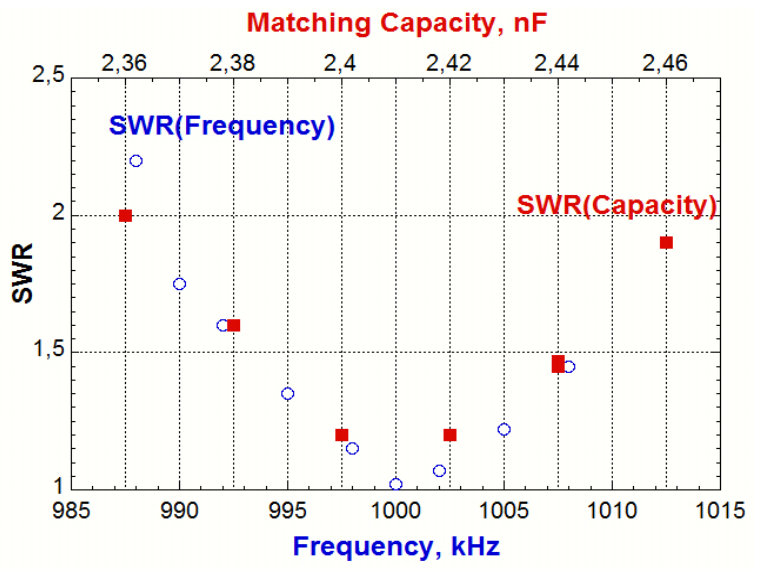

FIG.2. SWR at $0.5 \mathrm{~Pa}$ and $20 \mathrm{~kW}$ as a function of frequency (circles) and of the matching capacity at $1 \mathrm{MHz}$ (squares)

For the frequency matching a variation by $+/-12 \mathrm{kHz}$ is sufficient to cover the range of the allowed SWR. In Fig. 2 it is shown that the matching by frequency change is equivalent to that by changing the capacity. The advantage of frequency matching is that no access to the source is necessary, which in case of ITER is of particular importance, because the source is in radioactive environment.

The $\mathrm{H}_{\alpha}$ and $\mathrm{H}_{\beta}$ line intensities measured in the driver show the same dependence on source pressure and RF power with the transistor generator as with the oscillator.

\section{PLANAR COIL DRIVER}

The power losses correlated to the plasma expansion can be avoided by replacing the cylindrical driver by a flat insulator plate with a planar coil. Because the insulator has to stand the atmospheric pressure, plates of about 1.5 to 2 $\mathrm{cm}$ thickness are needed. The resulting large distance between coil and plasma leads to a poor coupling, as it is indicated in the left side of Fig. 3. A possible alternative suggested by Godyak ${ }^{5}$ is to concentrate the RF field of the coil in ferrites. This allows mounting the ferrites and the coil inside the vacuum onto a metallic flange (back plate), because the RF fields do not reach the metal surface and therefore cannot produce eddy currents (Fig.3). This allows a very compact design and enables to use thin insulators, which improves the coupling to the plasma.

Such a prototype source has been designed and manufactured at Augsburg University. The diameter of the cylindrical source chamber is $15 \mathrm{~cm}$ and the thickness of the Quartz insulator plate is $3 \mathrm{~mm}$.
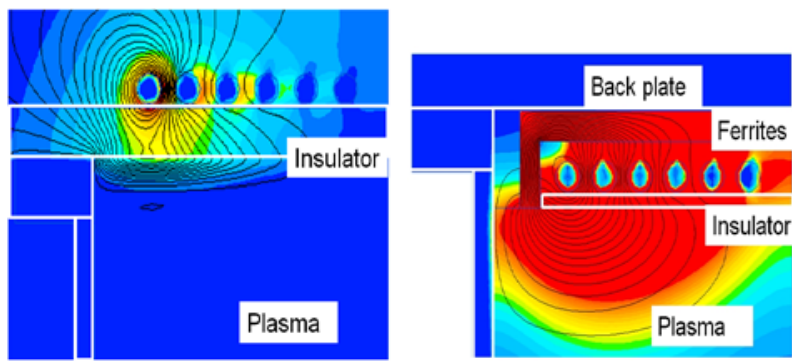

FIG.3. Sketch of the left half of planar coil sources, planar coil only (left) and tested source with field concentration by ferrites (right). FEM calculations of strength and field lines of the $\mathrm{B}_{\mathrm{RF}}$ field are indicated.

The insulator is protected from plasma erosion by a 1 $\mathrm{mm}$ thin Aluminum plate with radial slits (Faraday shield). A Teflon insulated RF coil of 5 turns is supplied in cw by a $2 \mathrm{MHz} / 2 \mathrm{~kW}$ solid state RF generator.

This source has been commissioned in Argon. The next step will be the operation in hydrogen and measurements of the emissions of the Balmer series and of the molecular Fulcher band in order to measure the plasma density and the dissociation degree.

\section{HELICON DISCHARGE}

Helicon discharges are known for their high efficiency arising from an RF coupling by wave heating. A proof of 
principle that an array of multiple sources can produce an uniform plasma has already been carried out in Argon ${ }^{6}$. The investigation of Helicon coupling in hydrogen and deuterium discharges is being carried out at a small laboratory setup (see Fig 4). It consists of a cylindrical discharge vessel made of quartz glass of $10 \mathrm{~cm}$ diameter and $40 \mathrm{~cm}$ length. An external magnetic field up to $12 \mathrm{mT}$ is generated by a pair of Helmholtz coils. A Nagoya type III antenna is utilized which can be replaced by a 5 turn solenoid. This allows distinguishing the impact of a magnetic field on an ICP discharge (better axial confinement) from that of Helicon wave coupling for efficient plasma production. As diagnostic method optical emission spectroscopy is applied with the line of sight along the axis of the discharge vessel.

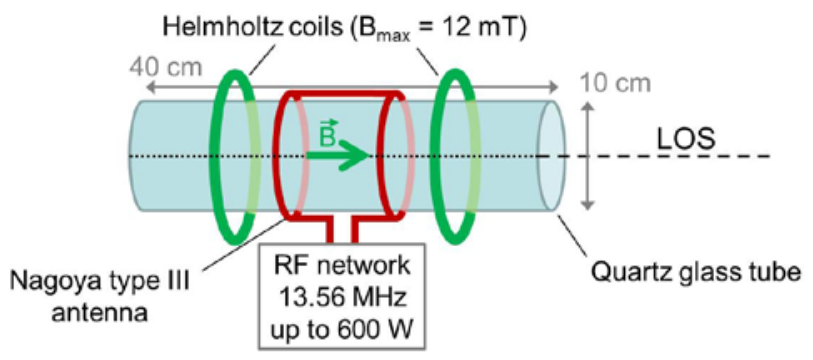

FIG.4. Sketch of the experimental setup for investigating Helicon discharges in hydrogen or deuterium.

In the ICP mode the emissions of the $\mathrm{H}_{\beta}$ line and of the Fulcher band increase slightly when the external magnetic field is switched on and reach a maximum at 2 $3 \mathrm{mT}$ (see Fig.5). This increase can be attributed to changed plasma profiles along the line of sight. By further strengthening the $\mathrm{B}$ field, the line emissions decrease strongly. Above $6 \mathrm{mT}$ the discharge jumps from inductive into capacitive mode.

With the Nagoya type III antenna the source is operated in the Helicon mode. At low B field the emission increases and reaches at around $3 \mathrm{mT}$ the so-called lowfield-peak, which is typical for Helicon discharges. Further increase of the B field results at first in a short decline, but above $4 \mathrm{mT}$ the emission increases again. This is not the case in the ICP mode, which proves that plasma confinement cannot be the reason - it is the Helicon coupling. The operation is possible at $0.3 \mathrm{~Pa}$ in hydrogen and - much better - in deuterium. A comparison of the emissivity of the two modes as shown in Fig. 5 is difficult, because changes of the radial profiles are not detected by the central single line of sight.

However, for the application as a driver of negative ion sources the required $\mathrm{B}$ fields might be too high and the low-field-peak seems therefore to be the more promising feature of the Helicon coupling. The goal of future experiments will be to achieve Helicon coupling with parameters closer to that of the drivers of negative ion sources. The frequency will be therefore reduced from

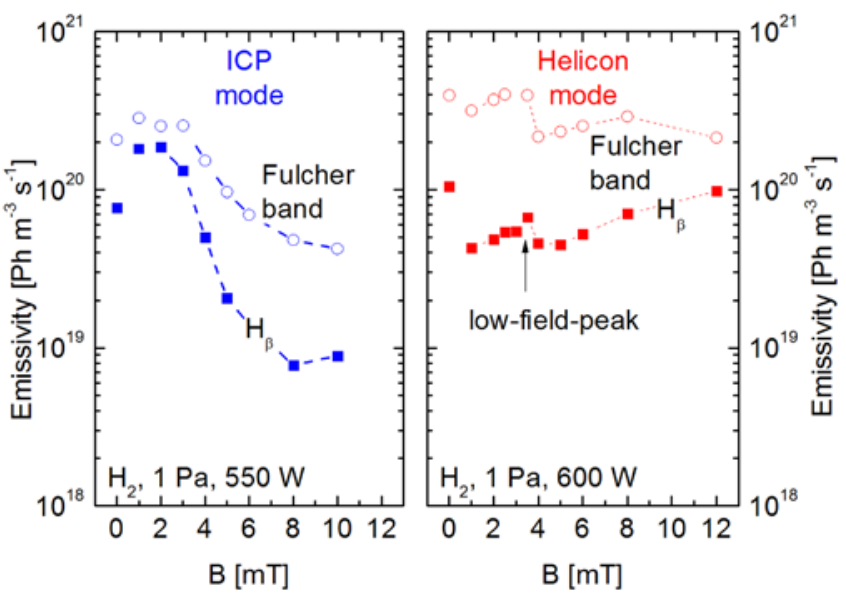

FIG.5. Emissivity of atomic $\mathrm{H}_{\beta}$ and molecular Fulcher band at varying magnetic field for ICP and Helicon operation.

13.56 to $2 \mathrm{MHz}$ and the diameter of the discharge tube will be enlarged to $20 \mathrm{~cm}$.

\section{SUMMARY AND CONCLUSION}

Efficiency and reliability of the power supply of RF driven negative ion sources can be improved substantially by replacing the oscillators by transistorized RF generators. In the ITER NBI the primary power could be reduced in this way for each source by almost $500 \mathrm{~kW}$. Further advantages are the simplification of the system due to the omission of a water cooling system and improved matching properties due to the stable frequency. A modified AM transmitter has shown in first tests the principal applicability of this generator type for plasma generation. The next steps will be to perform matching to different RF circuits and long-term reliability tests.

The investigation of new driver concepts, in which the plasma production is more efficient than in small cylindrical drivers, is under investigation. A planar ICP with a very compact design, enabled by the concentration of the RF field in ferrites, has been commissioned. Helicon coupling has been achieved in hydrogen and deuterium. The easiest way for the application for negative ion sources would be to use the low-field-peak. Future experiments will concentrate on lower frequency and larger source diameters.

${ }^{1}$ Hemsworth R, Decamps H, Graceffa J, Schunke B, Tanaka M, Dremel M, Tanga A, De Esch H P L, Geli F, Milnes J, Inoue T, Marcuzzi D, Sonato P and Zaccaria P 2009 Nucl. Fusion 49045006

${ }^{2}$ Fantz U, Franzen P, Kraus W, Berger M, Christ-Koch S, Falter H, Fröschle M, Gutser R, Heinemann B, Martens C, McNeely P, Riedl R, Speth E, Stäbler A and Wünderlich D 2009 Nucl. Fusion 49125007

${ }^{3}$ Kraus W, Fantz U, Franzen P, Fröschle M, Heinemann B, Riedl R and Wünderlich D, 2012 Rev. Sci. Instrum. 83 02B104

${ }^{4}$ Kraus W, Berger M, Fantz u, Franzen P, Fröschle M, Heinemann B, Riedl R, Speth E, Stäbler A, Rev. Sci. Instrum. 79 (2008) 02C108

${ }^{5}$ Godyak V A, Plasma Sources Sci. Technol. 20(2011) 025004

${ }^{6}$ Chen F, Torreblanca H, Physics of Plasmas16, 057102(2009) 